\title{
CALIDAD DEL AIRE EN TRES SITIOS DE ALTO TRAFICO VEHICULAR EN EL DISTRITO UNO DE LA CIUDAD DE MANAGUA
}

\section{AIR QUALITY IN THREE SITES WITH HIGH VEHICULAR TRAFFIC IN DISTRICT ONE OF THE CITY OF MANAGUA}

\author{
Norlan Javier Mejía Martínez ${ }^{1}$ \\ Diana Maribel Navarrete Pavón ${ }^{2}$ \\ Roberto José Dávila Altamirano ${ }^{3}$
}

(Recibido/received: 12-noviembre-2020; aceptado/accepted: 17-febrero-2021)

\begin{abstract}
RESUMEN: La presente investigación se realizó con el objetivo de evaluar la calidad del aire con respecto a tres contaminantes atmosféricos, material particulado menor o igual a 10 micras (PM10), dióxido de nitrógeno $\left(\mathrm{NO}_{2}\right)$ y ozono $\left(\mathrm{O}_{3}\right)$ en tres sitios de alto tráfico vehicular en el distrito uno de la ciudad de Managua, a través de la medición con equipos automáticos que permiten conocer el comportamiento de los contaminantes en tiempo real. Los monitoreos se realizaron entre octubre 2017 a enero 2018 con una frecuencia de dos muestreos por mes. En base a las concentraciones de contaminantes atmosféricos en el estudio se determinó que las concentraciones de PM10, dióxido de nitrógeno y ozono aumentan desde octubre 2017 (estación lluviosa) a enero 2018 (estación seca). Las concentraciones de PM10 y dióxido de nitrógeno no exceden el límite máximo permisible establecido en la NTON 05 012-02 durante los cuatro meses de medición, mientras que las concentraciones de ozono exceden la normativa de la NTON en los meses de noviembre y diciembre 2017 así como enero 2018.
\end{abstract}

\section{PALABRAS CLAVE:}

Calidad del aire, inmisiones, contaminantes atmosféricos, dióxido de nitrógeno, ozono, material particulado menor o igual a diez micras (PM10).

\footnotetext{
1 Responsable Laboratorio Físico Química Aguas Naturales Programa de Investigación, Estudios Nacionales y Servicios del Ambiente, PIENSA, de la Universidad Nacional de Ingeniería, UNI, norlan.mejia@piensa.uni.edu.ni

${ }^{2}$ Maestra en Ciencias Ambientales con mención en Gestión Ambiental, PIENSA-UNI, Nicaragua

${ }^{3}$ Maestro en Ciencias Ambientales, PIENSA-UNI, Nicaragua
} 
ABSTRACT: This research was carried out with the objective of evaluating air quality with respect to three atmospheric pollutants, particulate matter less than or equal to 10 microns (PM10), nitrogen dioxide $\left(\mathrm{NO}_{2}\right)$ and ozone $\left(\mathrm{O}_{3}\right)$ in three sites with high vehicular traffic in district one of the city of Managua through measurement with automatic equipment that allows knowing the behavior of pollutants in real time. The monitoring was carried out between October 2017 to January 2018 with a frequency of two samplings per month. Based on the concentrations of atmospheric pollutants under study, it was determined that the concentrations of PM10, nitrogen dioxide (and ozone increase from October 2017 (rainy season) to January 2018 (dry season). PM10 and nitrogen dioxide concentrations do not exceed the maximum permissible limit established in NTON 05 012-02 during the four months of measurement, while ozone concentrations exceed the NTON regulations in November and December 2017 as well like January 2018.

KEYWORDS: Air quality, emissions, atmospheric pollutants, nitrogen dioxide, ozone, particulate matter less than or equal to ten microns (PM10).

\section{INTRODUCCIÓN}

A través de los años debido al crecimiento poblacional y el incremento de las áreas urbanas e industriales, la calidad del aire se ha convertido en una problemática ambiental mundial, ya que dichas áreas generan inmisiones y provocan un impacto negativo en la salud de la población. Como consecuencia diferentes países y organizaciones, desde finales del siglo XIX han realizado estudios para obtener registros y evidencias del daño ambiental y del daño hacia la salud. Con el paso del tiempo, estos datos han aportado a la construcción de políticas y legislaciones ambientales enfocadas en reducir las concentraciones de los principales contaminantes atmosféricos.

Tanto es así que desde las dos últimas décadas del siglo XX se puede afirmar que "Hoy en día, son ampliamente reconocidos los problemas de contaminación ambiental, en particular del aire, que dan lugar a la acumulación de riesgos para la salud y el bienestar de la población", así lo aseveran Romieu (1995); Segala (1999); en Lacasaña-Navarro, Aguilar-Garduño y Romien (1999; 204). En las zonas urbanas, los vehículos son las principales fuentes de contaminación del aire debido a la quema de combustibles como gasolina y diésel. Según la Organización Mundial de la Salud (OMS, 2018) estima que la contaminación ambiental del aire, tanto en las ciudades como en las zonas rurales, fue causa de 4.2 millones de muertes prematuras en todo el mundo por año; esta mortalidad se debe a la exposición a partículas que causan enfermedades cardiovasculares y respiratorias, y cáncer.

En Nicaragua, los estudios de calidad del aire se han concentrado a las inmisiones de gases y partículas provenientes de los escapes de los automóviles, dejando a un lado lo referente a las emisiones de las industrias. El proyecto Aire Puro financiado por la Agencia Suiza para el Desarrollo y la Cooperación (COSUDE), fue ejecutado por la Fundación Suiza de Cooperación para el Desarrollo Técnico, (Swisscontact) en Nicaragua en el período comprendido entre 1996 a 2001. Este proyecto consistió en realizar mediciones de contaminantes atmosféricos tales como

El Higo Revista Científica / Volumen 11. No. 01, pp. 2-14 / junio 2021 
$\mathrm{NO}_{2}, \mathrm{O}_{3}, \mathrm{PM} 10$, PTS y acidez de la lluvia en siete estaciones ubicadas en la ciudad de Managua (La Subasta, Siete Sur, Recinto Universitario Simón Bolívar de la Universidad Nacional de Ingeniería, Colonia Centroamérica, Villa Libertad, Recinto Universitario Carlos Fonseca Amador de la Universidad Nacional Autónoma de Nicaragua y el Ministerio del Ambiente y los Recursos Naturales).

Entre los principales hallazgos del proyecto Aire Puro se determinó que las concentraciones de PM10 excedieron el valor guía de la OMS de $50 \mu \mathrm{g} / \mathrm{m}^{3}$ como promedio anual, Lo que evidenció que el PM10 representan un indicador de contaminación en la ciudad de Managua desde mediados de los años 1990, en el caso de dióxido de nitrógeno, no excedieron el valor guía de OMS de $40 \mu \mathrm{g} / \mathrm{m}^{3}$ como promedio anual, mientras que las concentraciones de ozono fueron fluctuantes, reportándose concentraciones mayores y menores al valor guía de OMS de $60 \mu \mathrm{g} / \mathrm{m} 3$, a lo largo del período de estudio. No existen datos horarios de dióxido de nitrógeno y ozono en la investigación del Proyecto Aire Puro, debido a que la metodología utilizada era de tubos pasivos, que consistía en la captación del contaminante a través de la adsorción en un sustrato químico por un período mayor a una hora, esta metodología imposibilitó determinar los aumentos de concentración con respecto al tráfico vehicular durante el día.

Como consecuencia de la información generada del proyecto, Dávila, quien también participó en el mismo, presentó en el año 2001 la investigación titulada: Estudio comparativo de contaminantes: $\mathrm{PM}_{10}, \mathrm{NO}_{2}$ y $\mathrm{O}_{3}$ durante 1997 y 1998 en tres puntos de alto tráfico vehicular en la ciudad de Managua, tanto en la estación seca como lluviosa. Los sitios seleccionados para esta investigación fueron: Colonia Centroamérica, Siete Sur y Gancho de Caminos. Se tomó en cuenta la influencia de las variables meteorológicas en la dispersión y transporte de los contaminantes. Como resultado de la investigación, Dávila determinó que las concentraciones de $\mathrm{NO}_{2}$ en los años no presentaron variaciones significativas entre estaciones de monitoreo y estaciones climáticas. Respecto a los promedios anuales de ozono en $1997(66 \mu \mathrm{g} / \mathrm{m3})$ y 1998 (76 $\mu \mathrm{g} / \mathrm{m} 3)$ superaron en más del $50 \%$ de las veces el valor guía establecido por OMS de 60 $\mu \mathrm{g} / \mathrm{m} 3$, también se determinó que existió diferencia significativa entre las concentraciones de la estación seca y lluviosa. Para las concentraciones de PM10 de ambos años no se presentó variación significativa, aunque al comparar las concentraciones por estación de monitoreo, el análisis de varianza demostró que sí existió diferencias significativas. Luego de esta investigación a la fecha no se ha encontrado evidencia de otros estudios relacionados con la contaminación del aire en Managua.

A nivel mundial, el registro de investigaciones evidencia la importancia de estudiar la calidad del aire debido a las consecuencias de la contaminación del aire en salud humana y ambiental. Es por ello que la presente investigación tiene por alcance evaluar el impacto en la calidad del aire en un período de cuatro meses, tomando en cuenta los contaminantes atmosféricos $\mathrm{PM}_{10}, \mathrm{NO}_{2}$ y $\mathrm{O}_{3}$ generados únicamente por el tráfico vehicular y el comportamiento de estos en la estación seca y lluviosa, tomando en cuenta la influencia de las variables meteorológicas y siendo comparados con los límites máximos permisibles de la Norma Técnica Obligatoria Nicaragüense de Calidad del Aire (NTON 05 012-02). 


\section{METODOLOGÍA}

De acuerdo a Fernández et al. (2017) una investigación puede tener una combinación de los diferentes tipos de estudios, pero nunca podrá ser básicamente de un solo tipo, sino que siempre tendrá elementos de alguno de los otros tipos de estudios. Es por esta razón que se presentan tres tipos de enfoques descriptivo, correlacional y explicativo considerando dos factores muy importantes como son el estado del aire en contaminantes atmosféricos y el enfoque a que se dio considerando el análisis de las variables y sus incidencias ambientales.

La investigación centra su análisis en determinar el comportamiento horario y mensual de los contaminantes en estudio ( $\mathrm{PM} 10, \mathrm{NO}_{2}$ y $\mathrm{O}_{3}$ ), así como la relación que existe entre las concentraciones de los contaminantes atmosféricos con respecto a las variables meteorológicas (temperatura, radiación solar, precipitación, humedad relativa, velocidad y dirección del viento), de tal manera La investigación describe una metodología integral para establecer una red sistemática y robusta de monitoreo de la calidad del aire en el distrito uno. La investigación fue desarrollada en cinco etapas secuenciales que se observan en el gráfico 1.

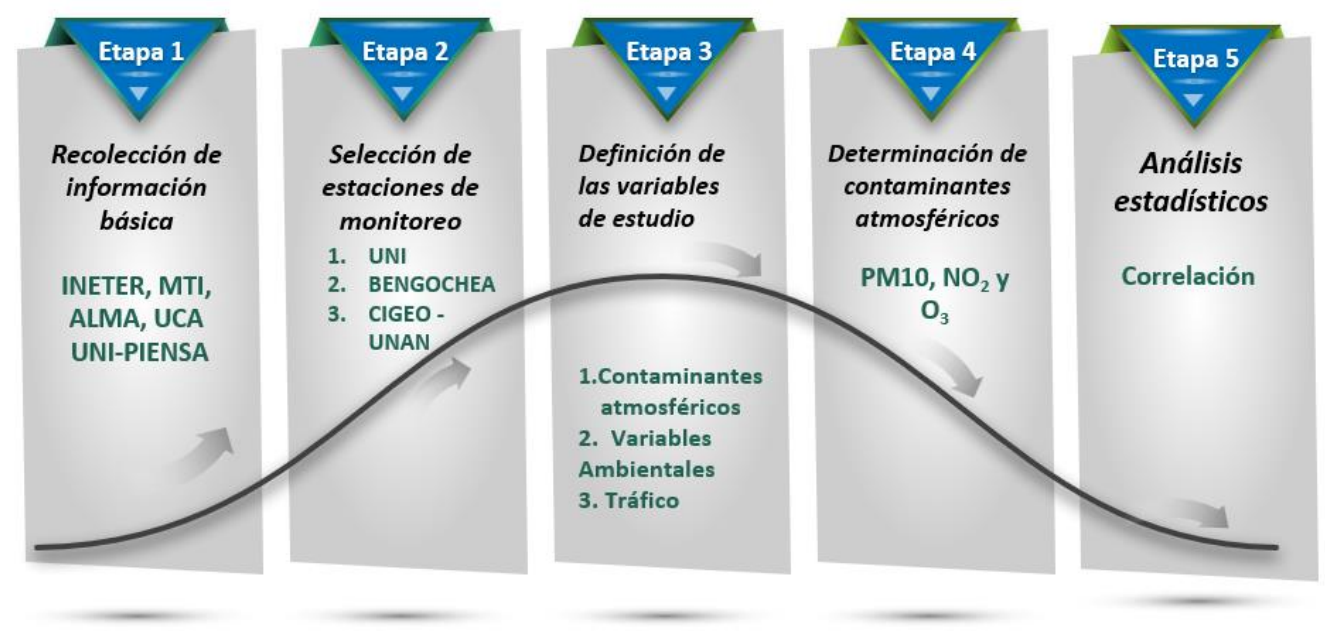

Gráfico 1: Esquema general de la metodología de la investigación

\section{Etapa 1: Recolección de información básica}

Se realizó la recolección de información de soporte, a través de la revisión bibliografía para conocer el estado de la calidad del aire en Nicaragua. Las fuentes consultadas son las instituciones del estado como:

Alcaldía de Managua (ALMA): se obtuvieron datos de aforos vehiculares del 2017 del departamento de vialidad, quienes realizan los aforos vehiculares e inventario vial de la ciudad de Managua, se obtuvieron los aforos de rotonda Rubén Darío, rotonda Roberto Terán y rotonda Universitaria. 
Instituto Nicaragüense de Estudios Territoriales (INETER): se obtuvieron datos de la Dirección General de Meteorología que es la instancia responsable de operar y explotar la Red Nacional de Estaciones Meteorológicas.

Estación Solar Julio López de la Fuente SJ de la Universidad Centroamericana (UCA): se obtuvieron datos de radiación solar.

El Ministerio del Ambiente y los Recursos Naturales (MARENA), se obtuvieron datos de los estudios en calidad del aire que se han realizado en Managua, así como las comunicaciones nacionales de Nicaragua ante la Convención Marco de Naciones Unidas de Cambio Climático.

El Programa de Investigación, Estudios Nacionales y Servicios Ambientales de la Universidad Nacional de Ingeniería (PIENSA-UNI) se obtuvieron antecedentes y resultados del proyecto aire puro, así como, los resultados de la investigación de Dávila (2000).

Esta etapa permitió explicar la importancia del problema de estudio y sus aportes a la sociedad. Se realizaron observaciones in situ, evaluaciones a los predios en que se encuentran previos a la realización de los monitoreos.

Etapa 2: Selección de estaciones de monitoreo

Para la realización de la investigación se utilizó como punto de partida las seis estaciones de monitoreo propuesta en el proyecto aire puro de 1996-2001 que estaban ubicadas en MARENA, Linda Vista, UNI, Colonia Centroamérica, Villa Libertad y Siete Sur. Sin embargo, de acuerdo a Dávila (2000) las estaciones que brindaron datos con mayor confiablidad fueron: Recinto Simón Bolívar (RUSB - UNI), Colonia Centroamérica y Siete sur.

De tal manera que para comparar los datos actuales (2017), con respecto a registro de 19971998, se tomó en cuenta una estación monitoreada por Dávila (2000) Laboratorios Bengoechea y una por el Proyecto Aire Puro estación UNI, añadiéndose CIGEO-UNAN, además que se consideraron los siguientes criterios:

Representatividad: las tres estaciones consideradas representan zonas representativas en cuanto a su tránsito promedio diario anual de acuerdo a cifras del MTI (2017). Cada día presentan un alto tránsito generado por ser zonas de entradas y salidas a la ciudad. Así como, zonas de tránsitos desarrollados por ser zonas comerciales de bienes perecederos y no perecederos.

Seguridad: en estos puntos se contaba con seguridad que permitió garantía de tener los equipos de muestreos sean dañados o robados por vándalos.

Condiciones meteorológicas y topográficas: no existen barreras naturales, ni artificiales, que obstruyan la difusión y transporte de los contaminantes en la dirección de los vientos, delante de los puntos seleccionados.

Distancia de los equipos de monitorio a la vía: las mediciones cercanas a la carretera generalmente se toman a una distancia mínima de 6-20 m del borde de la carretera, con el objetivo de reducir los impactos de los flujos de aire complejos en las mediciones (por ejemplo, turbulencia inducida por el vehículo, meandros en contra del viento). 
Etapa 3 Definición de las variables de estudio

Las variables de contaminación atmosférica que se consideraron para los tres sitios de monitoreo son: $\mathrm{PM} 10, \mathrm{NO}_{2}$ y $\mathrm{O}_{3}$.

La norma técnica obligatoria nicaragüense de calidad del aire (NTON 05 012-02) en su acápite seis, establece los lineamientos para el monitorio de los contaminantes atmosféricos:

El material particulado (PM10) se debe de realizar por 24 horas continúas, dos veces por semana por la técnica de volumen alto.

El dióxido de nitrógeno $\left(\mathrm{NO}_{2}\right)$ y Ozono $\left(\mathrm{O}_{3}\right)$ se deben realizar cada cinco minutos cada hora durante 8 horas continúas con instrumental automático.

De lo antes descrito en el acápite seis de la NTON 05 012-02, se debe aclarar que por motivos presupuestarios de la investigación y carga laboral del laboratorio de calidad del aire del UNIPIENSA, no se pueden realizar los monitoreos de PM10 dos veces por semana, porque se necesitarían más equipos de medición para lograr este fin, por lo tanto en la presente investigación se realizaron dos veces por mes y en el caso de dióxido de nitrógeno y ozono se realizaron por ocho horas dos veces por mes.

Debido a que a diversos autores han reportado que existe diferencia significativa entre la concentración de contaminantes en temporada de secas y de lluvias, siendo mayor la concentración de contaminantes en la temporada seca. En la ciudad de Managua de acuerdo a INETER (2000) se presenta un período lluvioso muy bien definido de mayo a octubre, con una disminución en los meses de julio a agosto debido al período de la canícula. A partir de noviembre se presenta el período seco, siendo el mes de febrero el más seco. Por esta razón, se abarcaron dos meses de la estación lluviosa y dos meses de la estación seca, los monitoreos se realizaron con un intervalo de 15 días aproximadamente, ejecutándose dos monitoreos por mes en cada sitio.

Las variables atmosféricas tomadas en consideración en la investigación fueron: temperatura, humedad relativa, precipitación, velocidad y dirección del viento, estas variables influyen en la velocidad con la que se efectúan las reacciones químicas atmosféricas. Por otro lado, la dispersión de estos contaminantes es influenciada por la velocidad y dirección de los vientos, así como la estabilidad que predomina en la atmósfera.

\section{Etapa 4 Determinación de los contaminantes atmosféricos}

Las muestras corresponden a las inmisiones de contaminantes atmosféricos ( $\mathrm{PM} 10, \mathrm{O}_{3}$ y $\left.\mathrm{NO}_{2}\right)$ emitidas a la atmósfera a través de la ignición de combustibles fósiles en los automotores en general que circulan en los tres sitios de muestreo.

Para el desarrollo de esta investigación, se decidió realizar una serie de rondas de muestreos en los tres sitios, el cual consistió en un monitoreo en el mes de octubre 2017, dos monitoreos en los meses noviembre 2017, diciembre 2017 y enero 2018, el intervalo entre los muestreos de noviembre 2017 a enero 2018 fue de 15 días aproximadamente. 
La medición de las concentraciones de Material Particulado se realizó en un intervalo de 10 segundos durante un período de veinticuatro horas, en el caso de Dióxido de Nitrógeno y Ozono, las mediciones se realizaron durante cinco minutos en una hora, en un período de ocho horas continúas, los datos fueron almacenados en la memoria interna del equipo EPAM 5000 y Aeroqual, siendo descargados a través de software al computador del Laboratorio de Calidad del Aire del PIENSA-UNI.

\section{Etapa 5. Análisis estadístico}

Los análisis estadísticos se realizaron con el software Microsoft Excel 2013.Se efectuó análisis de correlación de Spearman para determinar si existe correlación entre las concentraciones de los contaminantes en estudio y las variables meteorológicas.

El coeficiente de correlación de rangos de Spearman puede puntuar desde -1.0 hasta +1.0 , y se interpreta así: los valores cercanos a +1.0 , indican que existe una fuerte asociación entre las clasificaciones, o sea que a medida que aumenta un rango el otro también aumenta; los valores cercanos a -1.0 señalan que hay una fuerte asociación negativa entre las clasificaciones, es decir que, al aumentar un rango, el otro decrece. Cuando el valor es 0.0 , no hay correlación (Anderson et al., 1999)

\section{RESULTADOS Y DISCUSIÓN}

Como resultado de los siete muestreos realizados entre octubre 2017 y enero 2018 se determinó el comportamiento mensual de los tres contaminantes en estudio el cual se presenta a continuación:

\section{PM10}

El comportamiento mensual y estacional del PM10 se observa en la figura 1, durante octubre 2017 las concentraciones de PM10 son las de menor concentración en el período de estudio en las estaciones de la UNI y CIGEO, este comportamiento es debido a la presencia de precipitaciones que generan la deposición húmeda de las partículas hacia el suelo, disminuyendo las concentraciones en la troposfera, la estación Bengoechea no presenta este mismo comportamiento.

A partir de noviembre 2017 hasta enero 2018 las concentraciones de PM10 aumentan en las estaciones UNI y Bengoechea, esto es debido a la disminución de las precipitaciones que permite que las partículas en el suelo sean re suspendidas y transportadas a causa de la erosión que genera el viento cuando no hay humedad, mientras que las concentraciones de PM10 en la estación Bengoechea disminuyen desde octubre 2017 a enero 2018, este comportamiento es debido a la estructura vial de la rotonda Roberto Terán al tener una rotonda con paso a desnivel que permite que los automóviles permanezcan menos tiempo en la vía y las inmisiones de PM10 sean menores con respecto a las otras dos estaciones de monitoreo. 
Norlan J. Mejía M.; Diana M. Navarrete P.; Roberto J. Dávila A.

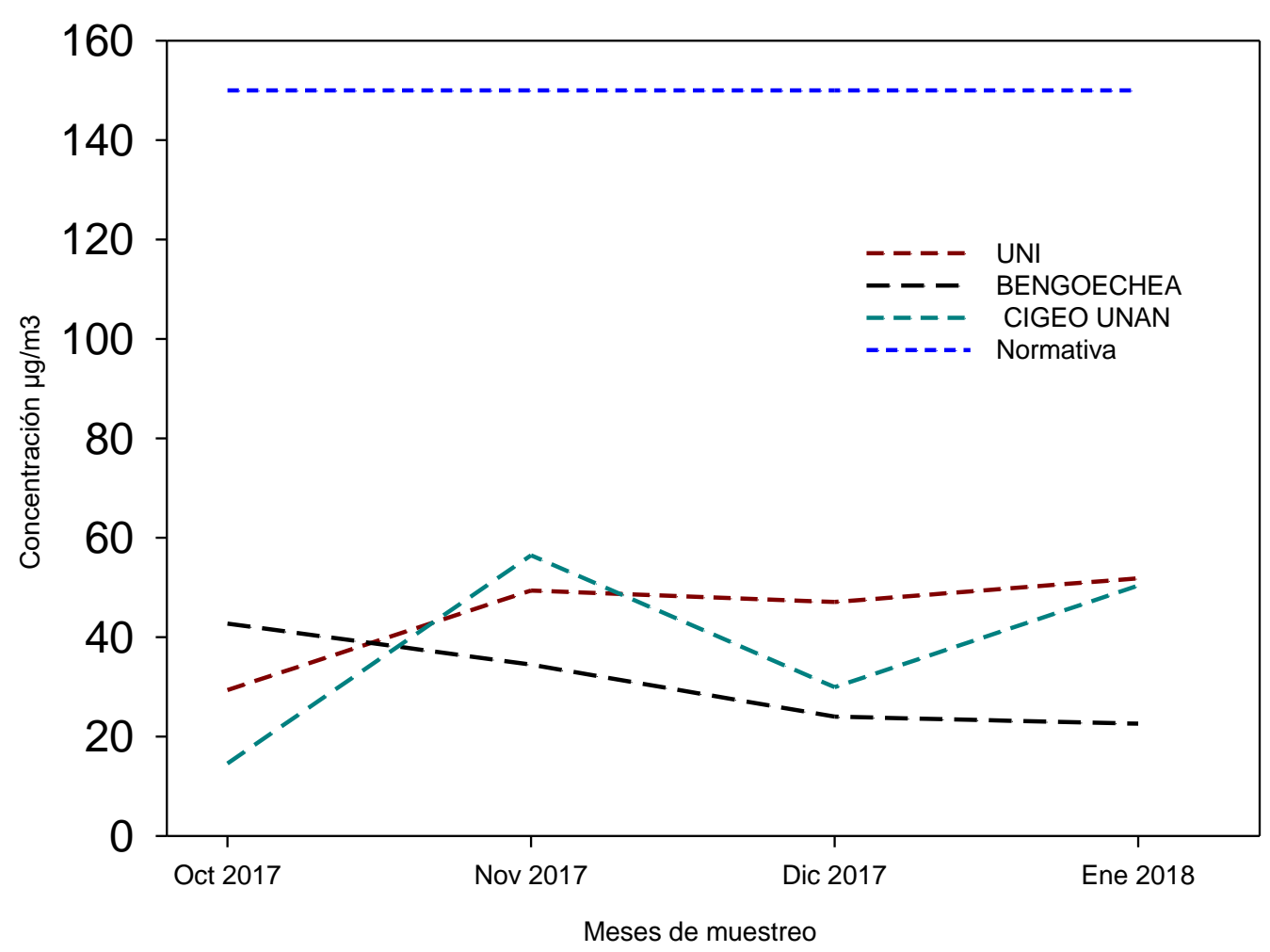

Figura 1. Comportamiento mensual de PM10

Al realizar el estadístico de correlación entre las concentraciones de PM10 y las variables meteorológicas se determinó que la velocidad del viento es la que presenta una correlación negativa considerable con el PM10 y por lo tanto cuando la velocidad del viento aumenta las concentraciones de PM10 disminuyen.

En ninguna de las tres estaciones se excede el límite máximo permisible de la NTON 05 012-02 de $150 \mu \mathrm{g} / \mathrm{m}^{3}$ para un período de 24 horas. Al comparar los resultados obtenidos con los valores guías de OMS (2005) y la Unión Europea (2008) para el contaminante PM10, de $50 \mu \mathrm{g} / \mathrm{m}^{3}$ para un período de 24 horas, se determina que la estación CIGEO excede los valores guía en noviembre $2017\left(56.47 \mu \mathrm{g} / \mathrm{m}^{3}\right)$ y enero $2018\left(50.35 \mu \mathrm{g} / \mathrm{m}^{3}\right)$, la estación UNI, en enero $2018(51.82$ $\mu \mathrm{g} / \mathrm{m} 3)$, mientras que la estación Bengoechea no los excede en ningún mes de monitoreo.

\section{Dióxido de nitrógeno}

Las concentraciones de $\mathrm{NO}_{2}$ durante el mes de octubre 2017 en la estación UNI presenta la menor concentración del período de estudio, mientras que las estaciones de Bengoechea y CIGEO tienen concentraciones mayores y similares, a como se observa en la figura 2. Este comportamiento es debido a las precipitaciones registradas durante octubre 2017 en el que el 
dióxido de nitrógeno reacciona con las gotas de lluvia formando ácido nítrico uno de los principales contribuyentes de la formación de la lluvia ácida.

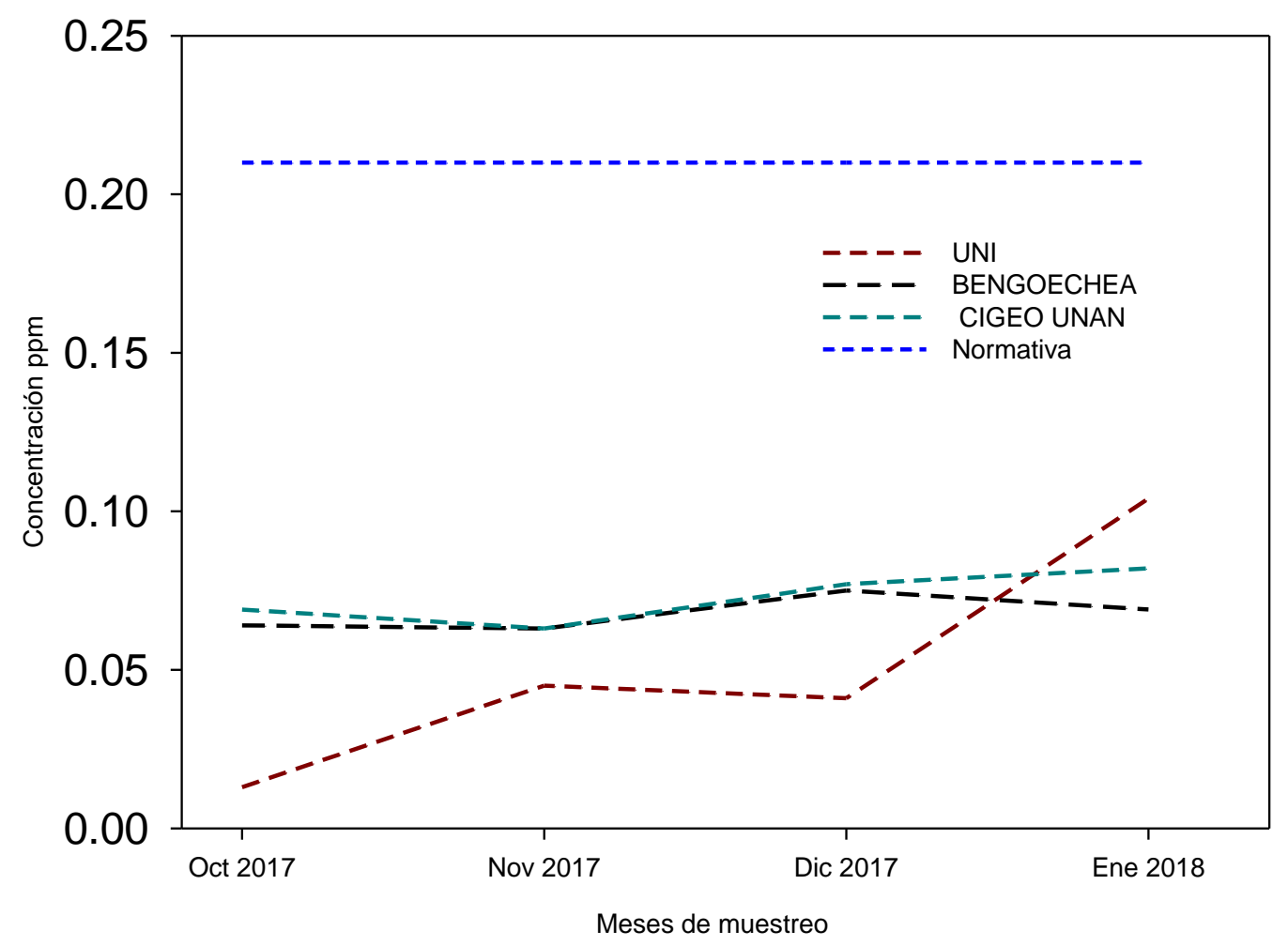

Figura 2. Comportamiento mensual de dióxido de nitrógeno

Desde noviembre 2017 a enero 2018 las concentraciones $\mathrm{NO}_{2}$ aumentan en las tres estaciones de monitoreo, obteniéndose las concentraciones más altas en enero 2018 , este comportamiento se debe principalmente a la disminución de las precipitaciones, característico de la estación seca en Managua, lo que permite que el contaminante persista en el aire ya que no reacciona con las gotas de lluvia aumentando su concentración.

Al realizar el estadístico de correlación entre las concentraciones de $\mathrm{NO}_{2}$ y las variables meteorológicas se determinó que la radiación solar, velocidad del viento y precipitación presentan una correlación positiva considerable con el $\mathrm{NO}_{2}$ y por lo tanto se comprueba que estas tres variables inciden en el transporte y dispersión de este contaminante.

En ninguno de los monitoreados realizados se excede límite máximo permisible de la NTON 05 012-02 de 0.21 ppm para una hora de medición. Con respecto a los valores guías de OMS (2005) y la Unión Europea (2008), para el contaminante $\mathrm{NO}_{2}$ es $200 \mu \mathrm{g} / \mathrm{m}^{3}$, valor medio para una hora de medición. Al comparar los resultados obtenidos en la presente investigación con las dos guías mencionadas anteriormente se determina que en ninguna de las estaciones de monitoreo se excede el valor guía. 


\section{Ozono}

En octubre 2017 la estación UNI presenta la menor concentración de ozono, seguido de la estación Bengoechea mientras que la estación CIGEO es la de mayor concentración a como se observa en la figura 3. En octubre se determinaron las menores concentraciones del período de estudio, este comportamiento es debido a las precipitaciones características de la estación lluviosa, debido a esto, el dióxido de nitrógeno, que es el contaminante precursor de la formación de ozono, reacciona con las gotas de agua de las precipitaciones formando ácido nítrico disminuyendo el $\mathrm{pH}$ de la lluvia y generando la lluvia ácida, debido a este fenómeno las concentraciones de ozono disminuyen.

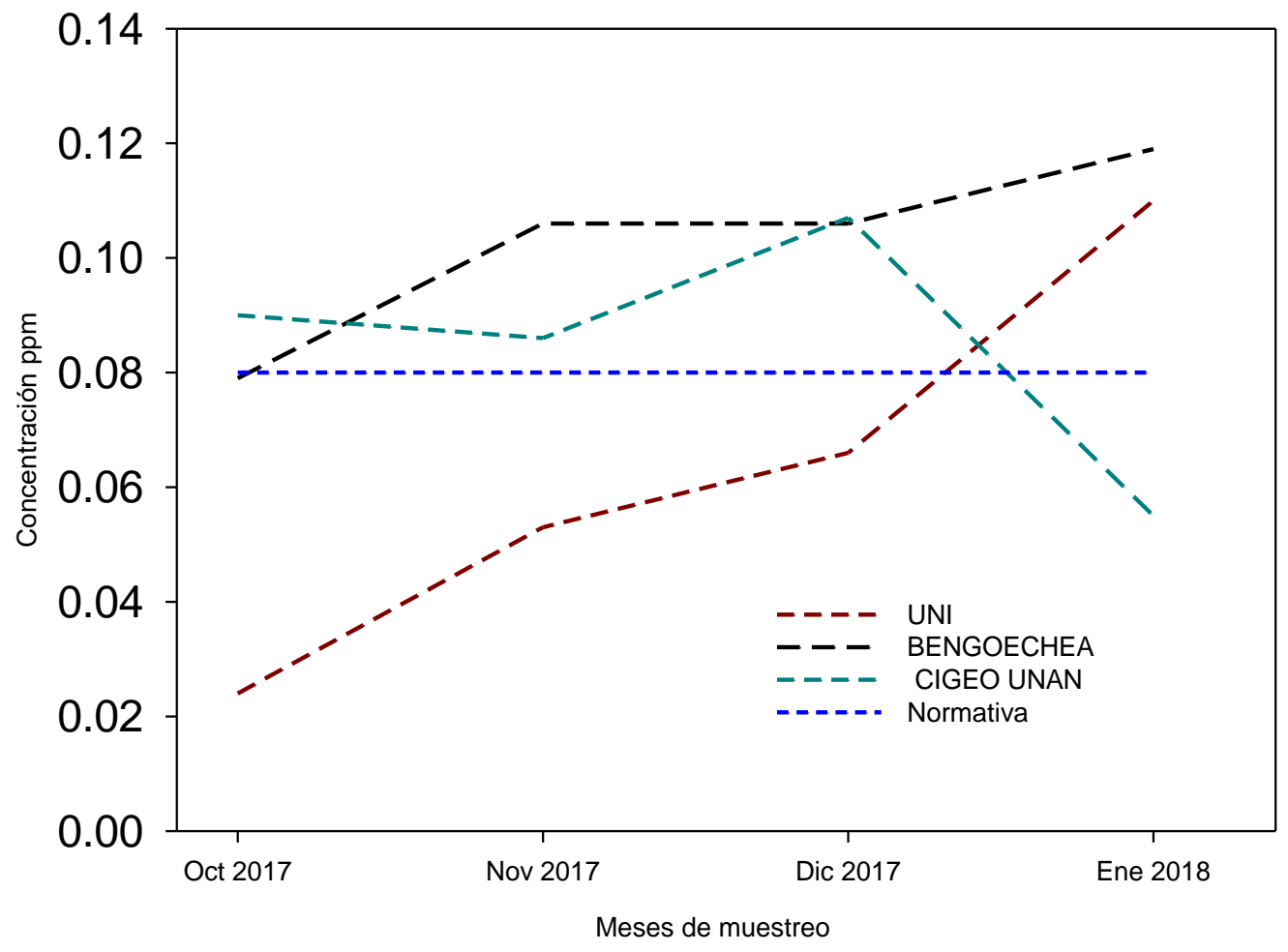

Figura 3. Comportamiento mensual de ozono

A partir de noviembre 2017 a enero 2018, a como se observa en el gráfico x, las concentraciones de ozono incrementan en las tres estaciones de monitoreo de calidad del aire, este aumento es debido a la disminución de las precipitaciones, menor nubosidad, mayor radiación solar e incremento de la temperatura, de esta manera el dióxido de nitrógeno, contaminante precursor del ozono, tiene las condiciones ideales para la formación de ozono troposférico.

Con respecto a la normativa nacional de calidad del aire, NTON 05 012-012, la estación UNI no excede el límite máximo permisible para ocho horas de medición de $160 \mu \mathrm{g} / \mathrm{m}^{3}$ en octubre, noviembre y diciembre del año 2017, excediéndolo únicamente en el mes de enero 2018. Las 
estaciones Bengoechea y CIGEO no exceden la normativa en el mes de octubre 2017, mientras que en noviembre y diciembre del año 2017 así como en enero 2018, exceden el límite máximo permisible de la normativa.

Al comparar los resultados obtenidos con respecto a los valores guías de OMS (2005) y la Unión Europea (2008), para el contaminante ozono es $100 \mu \mathrm{g} / \mathrm{m}^{3}$ y $200 \mu \mathrm{g} / \mathrm{m}^{3}$ respectivamente, para ocho horas de medición. Se determinó que la estación UNI no excede estas guías en el mes de octubre 2017, pero si los excede en los meses de noviembre 2017, diciembre 2017 y enero 2018. En el caso de las estaciones Bengoechea y CIGEO exceden estos valores guías en todo el período de estudio comprendido entre octubre 2017 a enero 2018.

Al realizar el estadístico de correlación entre las concentraciones de $\mathrm{O}_{3}$ y las variables meteorológicas se determinó que la radiación solar, velocidad del viento y temperatura presentan una correlación positiva considerable con el ozono, por lo tanto, se comprueba que estas tres variables inciden en la formación del ozono troposférico.

\section{CONCLUSIONES}

Se identificó el comportamiento mensual de los contaminantes atmosféricos, PM10, dióxido de nitrógeno y ozono, en las tres estaciones de monitoreo determinándose que las concentraciones de los mismos aumentan desde el período lluvioso (octubre a noviembre) hacia el período seco (diciembre y enero).

A través del cálculo de las correlaciones entre las concentraciones promedio día de los contaminantes y las variables meteorológicas en estudio se concluye que para PM10 existe una mejor relación con la velocidad del viento, mientras que para $\mathrm{NO}_{2}$ se presenta correlación las variables radiación solar, velocidad del viento y precipitación, mientras que ozono presenta mejor correlación con la temperatura, radiación solar y velocidad del viento.

Al contrastar las concentraciones de PM10, $\mathrm{NO}_{2}$ y $\mathrm{O}_{3}$ con la NTON de Calidad del Aire de Nicaragua NTON 05 012-02, se concluye que las concentraciones de PM10 y Dióxido de nitrógeno no exceden el límite máximo permisible de esta normativa entre octubre 2017 a enero 2018, mientras que las concentraciones de ozono exceden el límite máximo permisible de la normativa en los meses de noviembre y diciembre del año 2017, así como en enero 2018.

\section{REFERENCIAS}

Balladares, M. (2017). Proyecto Intersectorial Salud y Desarrollo Municipal MINSA/OPS/UNIPIENSA: Propuesta de monitoreo de aire en exteriores para la ciudad de Managua.

Dávila, R. (1997). Proyecto Aire Puro: Monitoreo de la calidad del aire de la ciudad de Managua (Informe Anual 1996).

Dávila, R. (1998). Proyecto Aire Puro: Monitoreo de la calidad del aire de la ciudad de Managua (Informe Anual 1997). 
Dávila, R. \& Jaen, R. (1999). Proyecto Aire Puro: Monitoreo de la calidad del aire de la ciudad de Managua (Informe Anual 1998).

Dávila, R. \& Jaen, R. (2000). Proyecto Aire Puro: Monitoreo de la calidad del aire de la ciudad de Managua (Informe Anual 1999).

Dávila, R. \& Jaen, R. (2001). Proyecto Aire Puro: Monitoreo de la contaminación del aire en la ciudad de Managua (Informe Anual 2000).

Dávila, R. (2001). Estudio Comparativo de contaminantes: PM10, NO2 y O3 durante 1997 y 1998 en tres puntos de alto tráfico vehicular en la ciudad de Managua (Tesis de maestría). Universidad Nacional de Ingeniería, Managua.

Dávila, R. \& Jaen, R. (2002). Proyecto Aire Puro: Monitoreo de la contaminación del aire en la ciudad de Managua (Informe Anual 2001).

Decreto del Reglamento General para el control de emisiones de los vehículos automotores de Nicaragua (No. 32-97), La Gaceta Diario Oficial No. 114 (1997).

European Enviromental Agency. Air quality in Europe - 2019 report. Recuperado de: https://www.eea.europa.eu/publications/air-quality-in-europe-2019

Lacasaña-Navarro, M., Aguilar-Garduño, C., \& Romieu, I. (1999). Evolución de la contaminación del aire e impacto de los programas de control en tres megaciudades de América Latina. Salud Pública de México, 41, (3) 203-215. Recuperado de: http://saludpublica.mx/index.php/spm/article/view/6150/7257

Ministerio del Ambiente y los Recursos Naturales. (2009a). Elementos de Diagnóstico de la Calidad del Aire y de su marco legal y gestión. Recuperado de:

http://www.sica.int/busqueda/busqueda_archivo.aspx?Archivo=odoc_71000_1_20072012.p df

Norma Técnica Obligatoria Nicaragüense de Calidad del Aire (NTON 05 012-02), La Gaceta Diario Oficial No. 211 (2002).

Organización Mundial de la Salud (2018). Calidad del aire y salud. Recuperado de: https://www.who.int/es/news-room/fact-sheets/detail/ambient-(outdoor)-air-quality-andhealth

Organización Mundial de la Salud (2006). Guías de Calidad del Aire de la OMS relativas al material particulado, el ozono, el dióxido de nitrógeno y el dióxido de azufre. Recuperado de: https://www.who.int/publications/list/who_sde_phe_oeh_06_02/es/

Swisscontact. (2001). Documentación de los sitios de muestreo; Monitoreo del Aire en Centro América. Recuperado de:

https://www.sica.int/busqueda/busqueda_basica.aspx?idCat=\&idMod=3\&ldEnt=2\%20\&Pag $=99$ 


\section{SEMBLANZA DE LOS AUTORES}

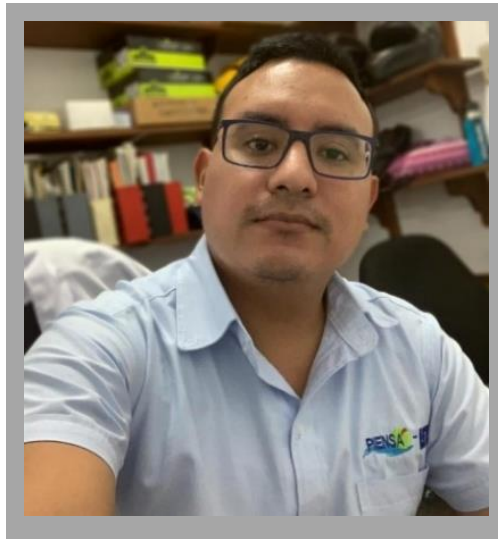

Norlan Javier Mejía Martínez: Es Licenciado en Química de la Universidad Nacional Autónoma de Nicaragua (UNANManagua), con Maestría en "Ciencias Ambientales con Mención en Gestión Ambiental" del Programa de Investigación Estudios Nacionales y Servicios del Ambiente de la Universidad Nacional de Ingeniería.

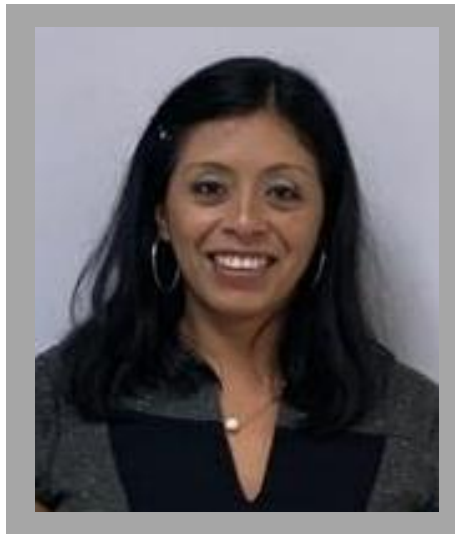

Diana Maribel Navarrete Pavón: Es Ingeniera industrial de la Universidad Nacional de Ingeniería, con Maestría en "Ciencias Ambientales con Mención en Gestión Ambiental" del Programa de Investigación Estudios Nacionales y Servicios del Ambiente de la Universidad Nacional de Ingeniería.

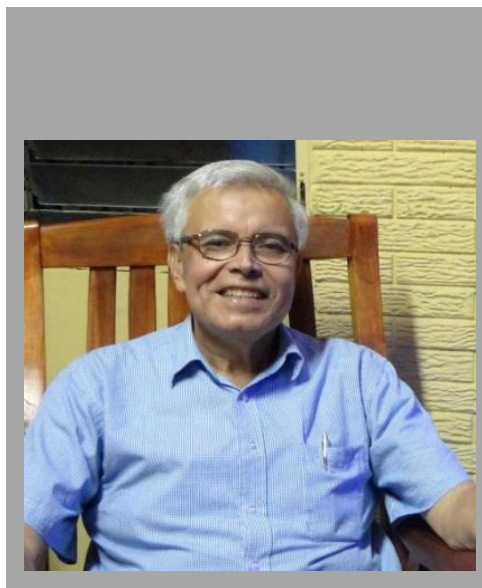

Roberto José Dávila Altamirano: Es Licenciado en Biología de la Universidad Nacional Autónoma de Nicaragua (UNANManagua), con Maestría en "Ciencias Ambientales" del Programa de Investigación Estudios Nacionales y Servicios del Ambiente de la Universidad Nacional de Ingeniería.

Jefe del Laboratorio de Calidad del Aire de PIENSA-UNI hasta septiembre 2010. Coordinador del Proyecto Aire Puro en CIEMAUNI 1996-2001. Docente de la asignatura de Contaminación del aire en PIENSA-UNI hasta febrero de 2020. Su línea de investigación fue la Calidad del aire, principalmente en la ciudad de Managua, Nicaragua. 